Marquette University

e-Publications@Marquette

Biomedical Engineering Faculty Research and

Publications

Biomedical Engineering, Department of

9-22-1999

\title{
Quantification of Pulmonary Arterial Wall Distensibility Using Parameters Extracted from Volumetric Micro-CT Images
}

Roger H. Johnson

Medical College of Wisconsin

Kelly Lynn Karau

Marquette University

Robert C. Molthen

Marquette University, robert.molthen@marquette.edu

Christopher A. Dawson

Medical College of Wisconsin

Follow this and additional works at: https://epublications.marquette.edu/bioengin_fac

Part of the Biomedical Engineering and Bioengineering Commons

\section{Recommended Citation}

Johnson, Roger H.; Karau, Kelly Lynn; Molthen, Robert C.; and Dawson, Christopher A., "Quantification of Pulmonary Arterial Wall Distensibility Using Parameters Extracted from Volumetric Micro-CT Images" (1999). Biomedical Engineering Faculty Research and Publications. 131.

https://epublications.marquette.edu/bioengin_fac/131 


\title{
Quantification of pulmonary arterial wall distensibility using parameters extracted from volumetric micro-CT images
}

\author{
Roger H. Johnson ${ }^{* 1,2,3.5}$, Kelly L. Karau ${ }^{1}$, Robert C. Molthen ${ }^{1.5}$ and Christopher A. Dawson ${ }^{1.4,5}$ \\ ${ }^{\prime}$ Department of Biomedical Engineering, Marquette University \\ Departments of ${ }^{2}$ Radiology, ${ }^{3}$ Biophysics and ${ }^{4}$ Physiology, Medical College of Wisconsin \\ ${ }^{5}$ Research Service, Zablocki VA Medical Center \\ Milwaukee, Wisconsin, USA
}

\begin{abstract}
Stiffening, or loss of distensibility, of arterial vessel walls is among the manifestations of a number of vascular diseases including pulmonary arterial hypertension. We are attempting to quantify the mechanical properties of vessel walls of the pulmonary arterial tree using parameters derived from high-resolution volumetric $\mathrm{x}$-ray CT images of rat lungs. The pulmonary arterial trees of the excised lungs are filled with a contrast agent. The lungs are imaged with arterial pressures spanning the physiological range. Vessel segment diameters are measured from the inlet to the periphery, and distensibilities calculated from diameters as a function of pressure. The method shows promise as an adjunct to other morphometric techniques such as histology and corrosion casting. It possesses the advantages of being nondestructive, characterizing the vascular structures while the lungs are imaged rapidly and in a near-physiological state, and providing the ability to associate mechanical properties with vessel location in the intact tree hierarchy.
\end{abstract}

Keywords: arterial tree morphometry, mechanical properties, distensibility, morphometric parameters, hypertension, vascular trees, micro-CT, $\mathrm{x}$-ray microtomography, conebeam, pulmonary physiology

\section{INTRODUCTION}

Pulmonary hypertension is a life-threatening condition which may be idiopathic or arise secondary to a variety of lung pathologies such as chronic obstructive pulmonary disease. Elevated arterial pressures in the lung could be caused by a chronic imbalance of vasconstriction and vasodilation factors, leading to a generalized or localized lumenal narrowing 1 . Altematively, dysfunctional platelet interaction with vessel walls could lead to intravascular thrombosis and arterial obliteration ${ }^{2}$. Studies utilizing histological and vascular corrosion casting methods have implicated changes in vascular wall biomechanics, lumenal narrowing and arteriolar obliteration as proximate causes of the elevated pulmonary arterial pressure ${ }^{3}$. Histologic and vascular casting methods are destructive and tedious and as a result have not been applied to questions such as the relative roles of lowered distensibility, generally reduced patency, and microemboli, and the levels in the pulmonary vascular tree hierarchy within which the biomechanical and morphological changes take place in pulmonary hypertension of different etiologies.

The available literature based on histological evidence often seems to suggest an inverse relationship between vessel medial wall thickness and distensibility. This is not necessarily the case, since a thinwalled vessel may become turgid at low pressure and exhibit low distensibility in response to increased intralumenal pressure, whereas a muscular vessel may be barely inflated at low pressure and show a high distensibility in response to pressure increases. A method capable of assessing distensibility nondestructively and allowing for subsequent spatial correlation with histomorphometric parameters like medial thickening and intimal proliferation would be a valuable tool for investigating the relationship between morphology and biomechanical properties.

*Correspondence: Email: JohnsonR@marquette.edu; tel: (414)288-7841; fax: (414)288-7938 http://perception.bien.mu.edw/molthen/Imaging/ImagingGroup.html 
A frequent method of investigation has been to inject the arterial tree with a radiopaque contrast material such as barium sulfate-gelatin mixtures (often prior to histologic sectioning or injection with polymeric casting material) and to obtain planar specimen radiographs of whole or partial lungs, if possible with some degree of magnification. For human lungs, large vessels appeared as distinct lines, but the evidence of diminution in caliber or obliteration or rarefaction of the small arteries was reported as an absence of "background haze" in the transmitted x-ray images of the opacified lungs ${ }^{4-7}$. A number of imaging studies on physiologically-maintained (fresh) intact lungs have employed planar x-ray images of the 3D branching arterial tree 8.9 . Though these studies provided valuable insights, the accuracy of quantitative metrics obtained from 2D images can suffer from problems associated with the unknown orientation of vessels with respect to the imaging system. The ability to nondestructively extract accurate dimensional measurements from high-resolution 3D images of lungs maintained in a physiological state would address many of the problems with previous techniques, and complement them in answering questions about how disease alters structure in ways detrimental to function. In this paper we report the development and application of 3D conebeam micro-CT techniques to the problem of determining pressure diameter relationships in the intact pulmonary arterial tree.

\section{MATERIALS AND METHODS}

\subsection{Lung preparation and data acquisition}

The micro-CT scanner, shown in Figure 1, was designed specifically for imaging intact small animal organs maintained in a physiological state ${ }^{10,11}$. Magnified cone-beam projection images are obtained by placing the specimen in close proximity to the source and situating the large-format imaging detector some distance away. The source-to-detector and source-to-specimen distances are chosen to produce a magnification (equal to the ratio of these distances) sufficient to enlarge the projection of the approximately $2.5-\mathrm{cm}$ rat lung to occupy the entire $17-\mathrm{cm}$ detector area. Whenever possible, the entire projection of the lung is captured in the detected image in order to avoid data truncation.

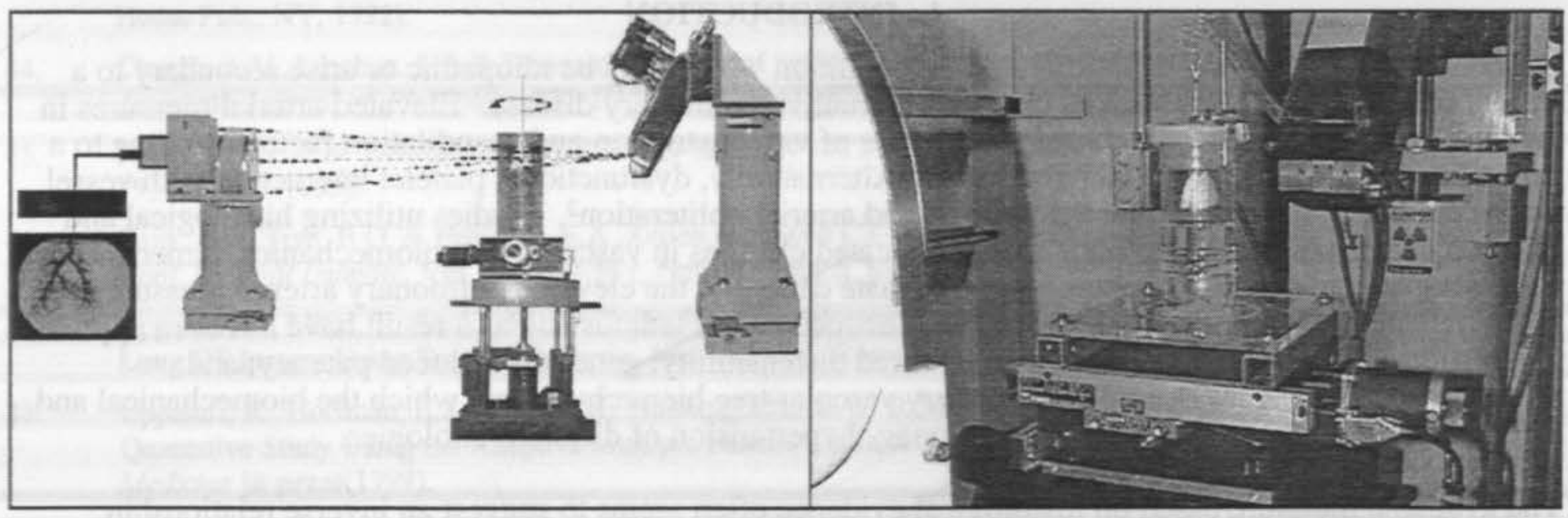

Figure 1: Schematic (left) and photograph (right) of the micro-CT scanner. The $\mathrm{x}$-ray source is on the right, the specimen stage and white rat lung in the center, and the detector on the left.

The scanner consists of a Feinfocus microfocal x-ray source and a Thomson 9-7-5" image-intensifier detector, operated in the 7-inch mode for rat lung imaging and coupled to a Silicon Mountain Design CCD camera using tandem optics (collimator lens plus objective lens). The source has a cylindrical tungsten anode and is operable over the 5- to $100-\mathrm{kVp}$ and 20-300 microamp ranges with elliptical focal spots as small as three by five microns. The detector, specimen stage and $\mathrm{x}$-ray source are mounted on an optical rail, with the optical axis and central fan of the conical x-ray beam contained in a horizontal plane through the system center. The specimen stage and image intensifier can both be moved along the optical axis to obtain the desired cone angle and magnification. A linear encoder monitors the stage and detector positions relative to the source. The 12 -bit, frame-transfer CCD camera has a $1024^{2}$ pixel array which, primarily due to data handling and throughput considerations, is normally read out at eight bits per pixel in 
$2 \times 2$ binning mode $\left(512^{2}\right.$ pixels). Frame averaging is used to produce a total $\mathrm{x}$-ray exposure of about one second per projection. The single-channel, $20-\mathrm{MHz}$ output of the CCD imager, coupled with its high fullwell capacity $(>300,000$ electrons/pixel) and low readout noise ( $<55$ electrons/pixel readout), provides a satisfactory compromise between data rate and noise without the problems, in the context of tomographic imaging, associated with matching the gains and offsets of multiple-output chips. For rat lung imaging the spatial resolution is limited by the $512^{2}$ projection image matrix, since the spatial resolution of the image intensifier at its input is 6.2 line pairs per mm. Images are digitized with an Imaging Technologies PCIbus frame grabber. A New England Affiliated Technologies high-tolerance micropositioning specimen stage allows specimens to be translated in three dimensions and rotated, providing the ability to acquire the desired transmitted $\mathrm{x}$-ray projections through the specimen. Stage position and motions and camera operations are under the control of two Pentium PC's.

To obtain projection data sets of the excised lung, the rat was anesthetized and heparinized, then exsanguinated. The trachea and pulmonary artery were cannulated and the lungs removed from the chest. The lung vasculature was cleared of blood by flushing with a physiological saline solution, and the lungs suspended by the tracheal and pulmonary arterial cannulas from the top of a radiolucent plastic cylinder. A brominated contrast agent (perfluorooctyl bromide) was introduced through the arterial cannula at constant and selectable pressure. When introduced into the pulmonary artery, this contrast agent fills only the arterial tree due to the surface tension at the perfluorocarbon-aqueous interface. The airway pressure was held constant at $6 \mathrm{~mm} \mathrm{Hg}$ throughout the imaging procedure. The rat lung was imaged using the parameters given in Table 1. Four separate sets of projection data were taken at different intra-arterial contrast agent pressures spanning the physiological range. Pressures were referenced to the center of the scanner, which was also the center of the lung.

Table 1: Data acquisition and reconstruction parameters

\begin{tabular}{|l|l|l|}
\hline Parameter & Unit & Value \\
\hline airway pressure & $\mathrm{mm} \mathrm{Hg}$ & 6.0 \\
\hline arterial pressure & $\mathrm{mm} \mathrm{Hg}$ & $5.4,12.0,21.0,30.0$ \\
\hline tube voltage & $\mathrm{kVp}$ & 83 \\
\hline tube current & microamps & 24 \\
\hline source-to-detector & $\mathrm{cm}$ & 85.2 \\
\hline source-to-center & $\mathrm{cm}$ & 26.6 \\
\hline cone (half-) angle & degrees & 5.9 \\
\hline magnification & & 3.2 \\
\hline max linear dimension of object & $\mathrm{cm}$ & 2.5 \\
\hline II mode & inch/cm & $7 / 17.8$ \\
\hline number of views ( @ $1^{\circ}$ angular increments) & & 360 \\
\hline frames averaged per view & & 30 \\
\hline data acquisition time & minutes/volume & 10 \\
\hline acquisition matrix & pixels & $512^{2}$ \\
\hline reconstruction matrix & voxels & $497^{3}$ \\
\hline reconstruction volume & $\mathrm{cm}^{3}$ & 3.5 \\
\hline
\end{tabular}

\subsection{Image reconstruction}

Projection preprocessing has been described in detail elsewhere ${ }^{10,12}$. Briefly, it consists of polynomial unwarping to correct the pincushion and other distortions due to the intensifier imaging chain based on an image of a ball bearing (BB) grid phantom, followed by cropping the images to center the projection of the 
rotation axis (located with the aid of a sinogram). Finally, the nonuniform illumination intensity is accounted for by flood field division.

We reconstruct 3D image volumes from the processed projections using the Feldkamp conebeam algorithm $(\mathrm{FDK})^{13}$. Conebeam scanning is the most rapid and dose-efficient method for data acquisition, allowing sufficient data for a volumetric reconstruction to be collected in the time it formerly took to collect data for a single slice. As long as the cone (half-) angle is kept small (less than about $10^{\circ}$ ), the distortions and inexact nature inherent in the algorithm are not a problem for structural measurements. Instead of having to produce reformatted sagittal and coronal slices, as is the case in slice- by-slice fan-beam scanning, conebeam CT provides an isotropic reconstructed volume directly. Projection preprocessing and image reconstruction are performed on an SGI O2 workstation with a $175-\mathrm{MHz}$ R10000 processor, 1 GB of memory and 1-MB secondary cache. Reconstruction of a $500^{3}$ isotropic volume takes about 30 hours, but tests of one slice can be run in less than five minutes and eleven $500^{2}$ slices can be reconstructed in about two hours. In the interest of throughput for rapid phenotyping studies, an important objective of our current work is to speed up the reconstruction process (primarily the backprojection and, to a lesser extent, the convolution filtering) by parallelizing the algorithm where possible and taking advantage of the graphics hardware on the SGI platform to perform the computationally intensive multiply-accumulate operations.

\subsection{Morphometric measurements and data analysis}

Due to the complex structure of the pulmonary arterial tree which, in the case of the rat, contains several hundred thousand vessel segments, along with the fact that fully-automated computer analysis methodologies have not yet reached functional maturity, it is impossible to characterize every segment in the tree. We have therefore proposed and developed a method for data reduction based upon characterization of the single longest pathway--the main trunk--connecting the inlet to the smallest resolvable vessels at the periphery of the lung. Since this principle pathway contains vessels of every size-in every generation or order--present in the tree structure, it might be argued that for diffuse diseases such as pulmonary hypertension it is representative of the tree as a whole. For example, if vessels in a certain size range were implicated in pulmonary hypertension due to decreased distensibility or lumenal narrowing, image-based characterization sensitive to biomechanical properties and dimensions of the principle pathway would reveal the involved segments. Such a finding might be extrapolated to the entire tree or guide the selection of further segments or pathways for quantitative characterization.

This approach is based on the concept that the self-similar nature of the pulmonary arterial tree is such that in a statistical sense all portions of the tree downstream from a vessel of a given diameter are quantitatively similar. Taking this point of view, if one were to measure the diameter (D) of the larger trunk vessel and the smaller branch diameter at each bifurcation, and the distances between bifurcations off the main trunk, beginning at the pulmonary artery and ending with the terminal artery, the whole tree would be characterized. The measured segment diameters and distances can then be represented, for example, as graphs of diameter vs. total distance from the inlet. In the case of linear relationships, the slopes and intercepts of such graphs might serve as functionally relevant parameters in a morphometric summary of the tree structure. For nonlinear relationships the form of the fit may reveal functional differences between different tree structures. We have demonstrated that these parameters are sensitive to interspecies differences ${ }^{11}$, and believe they hold promise as indicators of functional impairments such as those seen in pulmonary hypertension.

To extract the diameter measurements for this study, the reconstructed gray-level data were analyzed interactively by following the longest pathway through the arterial tree, starting at the pulmonary arterial inlet and proceeding down to the smallest resolved vessels. Since most segments in the major pulmonary arterial trunk (the longest pathway) were close to vertical in orientation, the operator, using ScionImage (the PC version of NIH Image), would "fly through" a stack of horizontal transaxial slices in which obliquely-intersected vessel cross sections were elliptical. At each branch point the $3 \mathrm{D}$ coordinates of the bifurcation were identified as the last picture element joining two daughters before they divided. 
Specification of bifurcations in this manner using animated interaction with the data volume is simple and reproducible. The diameter of each segment of the longest pathway was measured at its midpoint by accessing and analyzing the slice equidistant between each pair of contiguous bifurcations. Segment diameters were measured according to the full-width at half-maximum (FWHM) criterion applied to line scans extracted along the minor axis of the elliptical vessel segment cross sections. Segment lengths were computed as the 3D distances between points at the center of the trunk lumen in transaxial slices containing contiguous bifurcations.

We define a principal pathway as any continuous path through the tree consisting of the set of connected arterial vessel segments defined by following the daughter branch with the larger diameter at each bifurcation as one proceeds toward the lung periphery along the pathway. The analysis of the distance (length) and diameter data involves fitting equations to the morphometric measurements extracted from the principal pathway which begins at the inlet. We have previously shown that for the rat the relationship between segment diameters and distance from the inlet is linear, meaning that the principal pathway can be viewed as a tapered cone with straight walls ${ }^{11}$. The slope and intercept of the regression line, as well as the coefficient of variation (variability of the data) about the line, are morphometric parameters describing elements of the structure of the tree. The slope, for example, quantifies the taper of the conical principle pathway.

For any individual vessel segment, plotting the diameter as a function of pressure reveals information about the distensibility over the investigated pressure range. For vessel segments remote from the scanner (lung) center, the nominal pressures would need to be corrected for the height of contrast agent actually seen at each location in the lung.

\section{RESULTS}

Figure 2 shows fully-processed projection images of a normal fawn-hooded rat lung from the same view angle at four different intra-arterial pressures. Figures 3 and 4 show surface-shaded renderings of segmented reconstructed image volumes, the former produced by a relatively high global threshold and the latter by a seeded region growing segmentation method. Figure 5 is a graph, for the principle pathway through the lung, of vessel segment diameter vs. distance from the inlet, each of the four lines representing the data at one of the four pressures investigated, produced with data from the lung depicted in the prior figures. Figure 6 shows the diameter vs. pressure relationship for one medium-caliber vessel segment at the center of the lung.

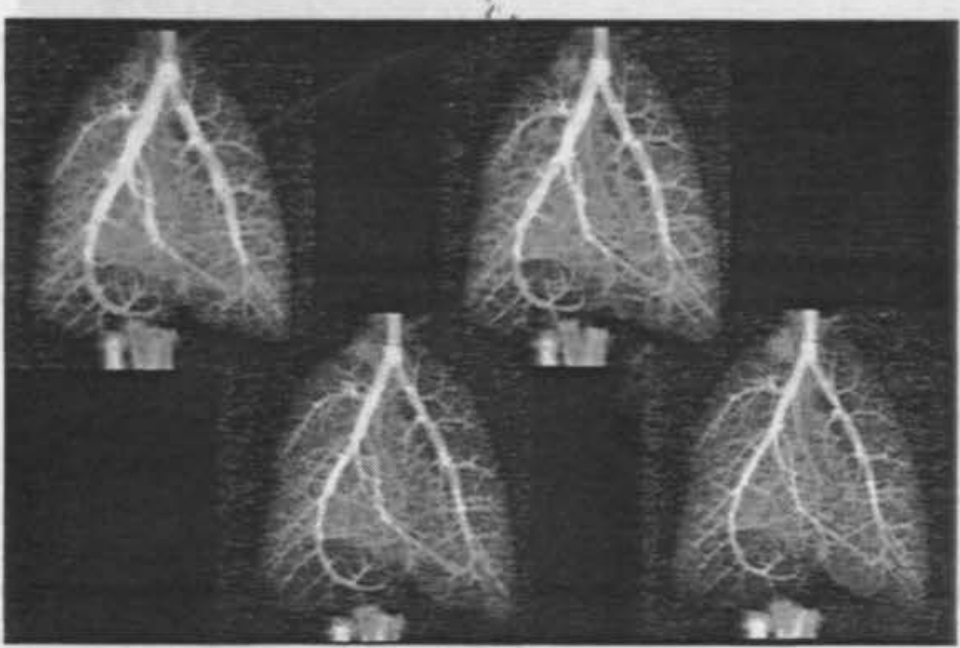

Figure 2: Projections of the rat lung at the four pressures indicated in Fig. 3.

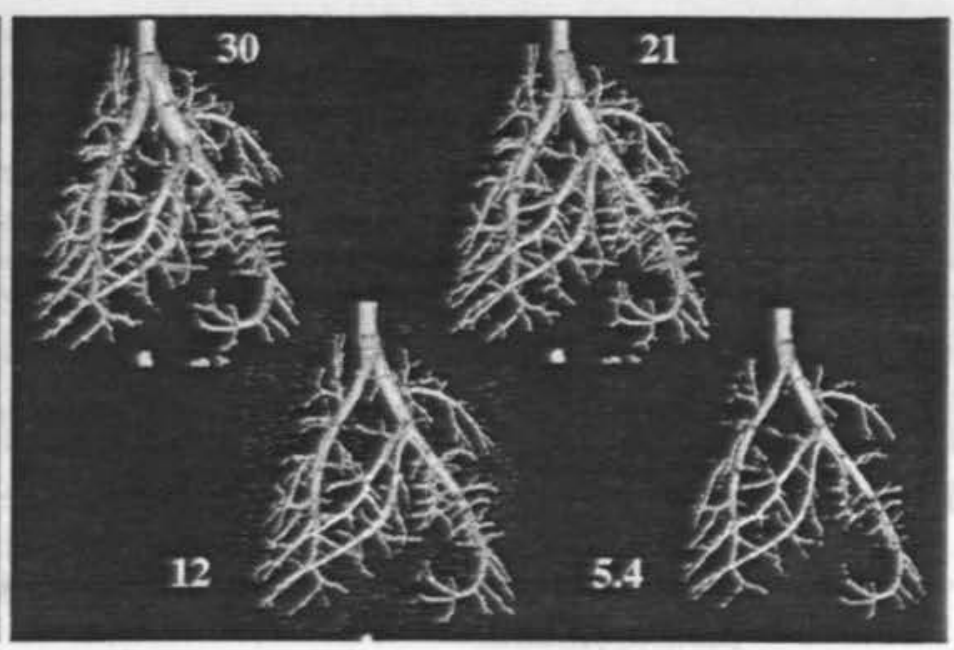

Figure 3: Globally-thresholded renderings of the rat lung at the four pressures indicated in units of $\mathrm{mm} \mathrm{Hg}$. 


\section{DISCUSSION}

The distension of the arterial tree with increased intraluminal pressure is clearly qualitatively evident, both from the projection images of Figure 2 and from the renderings of Figures 3 and 4 . Although all measurements reported were extracted from the as-reconstructed gray-level image data, the renderings assist in identifying the longest pathway through the tree and can be useful in appreciating other aspects of the 3D structure. We have investigated a number of segmentation methods, since segmented image volumes are useful for quantitative measurements of parameters like total arterial tree volume, and constitute the first step in a number of analysis and data reduction techniques such as skeletonization. We have found that global thresholding methods, one result of which is shown in Figure 3 , fail to faithfully differentiate vessel from non-vessel voxels, primarily due to volume averaging and artifacts inherent in the reconstruction. Histogram-based adaptive thresholding methods have also failed in our hands, due to difficulties in choosing appropriately robust features of the histogram distribution. The seeded region growing method illustrated in Figure 4 performs better in the sense that small vessels can be retained in the tree structure without incurring the unacceptable background noise penalty which is unavoidable using global or histogram-based locally adaptive thresholds.

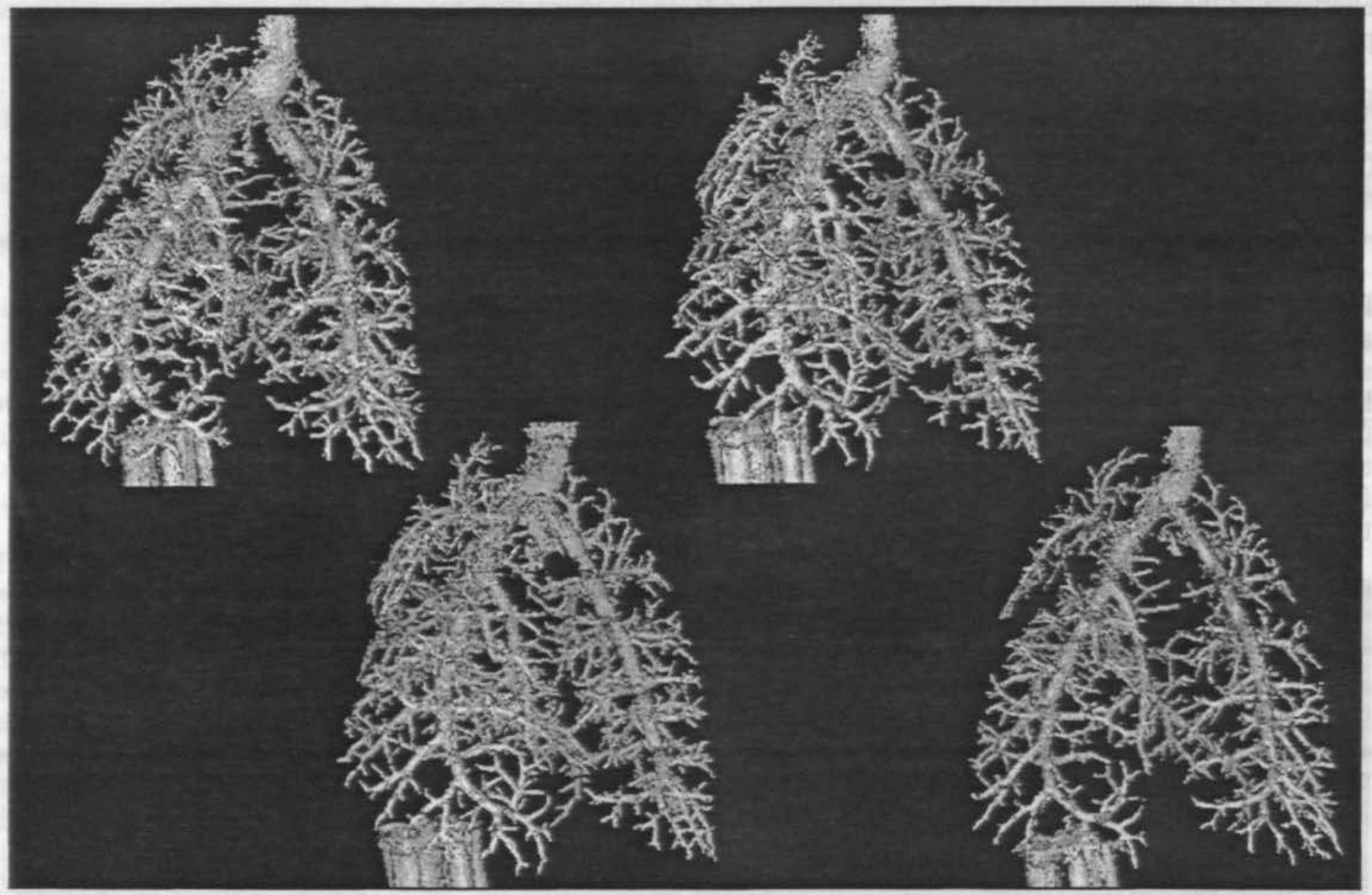

Figure 4: Renderings of the rat lung at the same pressures as Figures 2 and 3, produced using a seeded region growing segmentation method. The structure seen at the bottom of each image field is a contrast agent-filled phantom of tubes of known internal diameter used to calibrate diameter measurements.

The graph of Figure 5 is the primary result from this preliminary study. It shows quantitatively how the intercept and slope of the diameter vs. distance relationship increase with arterial pressure. Statistically, the linear fits are as appropriate as any other standard method for modeling the data, and the slope quantifies the average taper of the conical principle pathway through the tree. The consistency across the 
four pressures of the local variations in the degree of taper of the trunk is markedly apparent as well. For example, when the residuals associated with the $30 \mathrm{~mm} \mathrm{Hg}$ data were plotted against those of the $21 \mathrm{~mm}$ $\mathrm{Hg}$ data (plot not shown), the r-squared value of 0.7 indicates that most of the variability about the regression line is attributable to real variations in taper, not to noise such as imprecision in diameter measurements.

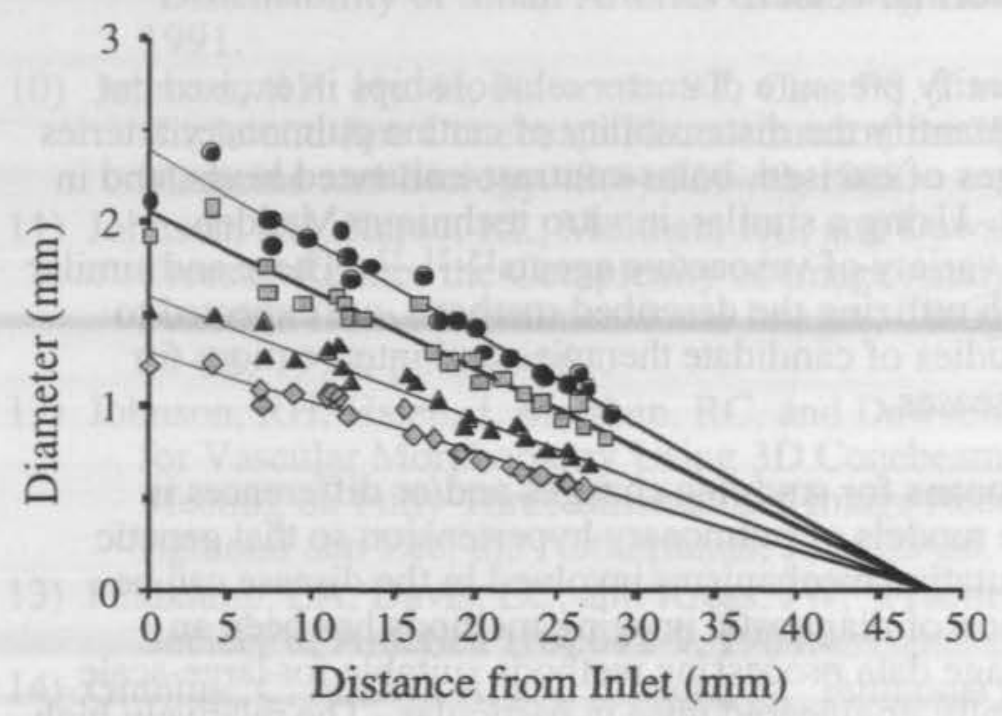

Figure 5: Diameter vs. distance from inlet for main trunk at four different intra-arterial contrast agent pressures.

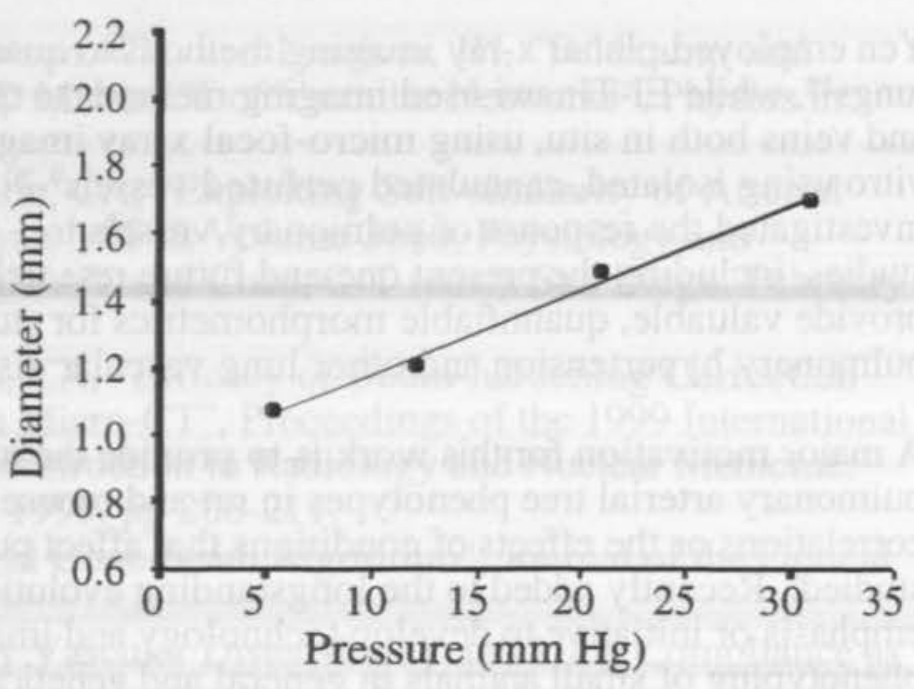

Figure 6: Diameter vs. pressure for a vessel near the center of the rat lung.

A number of previous studies have sought information on the distensibility of the pulmonary vasculature as a whole or the distensibility or elasticity of the various segments in the vascular tree. Distensibility has been variously used to denote the ratio of the change in volume of a vascular segment (or collection of segments, including the entire pulmonary arterial tree) to the change of intravascular pressure ${ }^{14}$, a concept properly associated with the term compliance, or to denote the fractional diameter change per unit change in intravascular pressure (a more accessible quantity in imaging experiments where individual diameters may be measured over a range of pressures). We have adopted the convention of Madden, El-Tinawi and others who define distensibility, $\beta$, as the slope of the diameter vs. pressure relationship and the distensibility coefficient, $\alpha$, as $\beta / D_{0}$, where $D_{0}$ is the diameter measured at an intravascular pressure of zero 9.15 . The diameter vs. pressure relationship, depicted for a typical medium-sized artery in Figure 6, can be used to calculate $D_{0}, \beta$, the change in diameter (microns) per Torr, and $\alpha(\% /$ Torr). In this case they were equal to 922 microns, 25 microns/Torr and $2.63 \%$ /Torr, in excellent agreement with previously reported values for canine pulmonary arteries ${ }^{9}$.

Giuntini performed an elegant in vivo, clinical experiment in which pulmonary vascular distensibility (PVD) represented the sum of the distensibility of all the vessel segments in the tree ${ }^{14}$. He found the PVD measured after an $1100 \mathrm{ml}$ dextran infusion was $5 \mathrm{ml}$ per $\mathrm{m}^{2}$ per $\mathrm{mm} \mathrm{Hg}$, resulting from a $16 \%$ increase in pulmonary blood volume accompanied by a $129 \%$ increase in mean pulmonary intravascular pressure. This very low distensibility appeared to refute the previously-held notion that the pulmonary vascular bed in vivo was a highly distensible system capable of passively accommodating large quantities of blood for example upon acute increases in total blood volume. But after atropine injection, this distensibility increased five-fold, indicating that active mechanisms may be responsible for the low in vivo distensibility of the healthy human lung.

Engelberg and DuBois showed, in the isolated rabbit lung, that various arterial segments have differing distensibilities, suggesting the value of localized measurements for various vessel diameter regimes ${ }^{16}$. 
Drangova used a laboratory, fan-beam CT system to obtain measurements of the elasticity of normal and aneurysmic abdominal aorta imaged over a range of five transmural pressures ${ }^{17}$. They reported a tangential or incremental modulus of elasticity, similar to Young's modulus, but deemed appropriate for nonlinear elastic materials such as arterial walls ${ }^{18}$. They found that a six-fold decrease in elastin, demonstrated histologically in the aneurysmic arterial wall, caused a 275 -fold increase in the incremental modulus at high transmural pressure relative to the normal vessel.

Yen employed planar $\mathrm{x}$-ray imaging methods to quantify pressure diameter relationships in excised cat lungs ${ }^{i 9}$, while El-Tinawi used imaging methods to quantify the distensibility of canine pulmonary arteries and veins both in situ, using micro-focal x-ray images of excised, bolus-contrast-enhanced lungs, and in vitro using isolated, cannulated perfused vessels 9,20 . Using a similar, in vitro technique Madden investigated the response of pulmonary vessels to a variety of vasoactive agents ${ }^{15,21,22}$. These and similar studies, including the present one and future research utilizing the described methods, are expected to provide valuable, quantifiable morphometrics for studies of candidate therapies and interventions for pulmonary hypertension and other lung vascular diseases.

A major motivation for this work is to provide the means for studying changes and/or differences in pulmonary arterial tree phenotypes in rat and mouse models of pulmonary hypertension so that genetic correlations or the effects of conditions that affect putative mechanisms involved in the disease can be studied. Recently added to the longstanding evolution of diagnostic imaging methods has been an emphasis or initiative to develop technology and image data processing methods suitable for large-scale phenotyping of small animals in general and genetically-engineered mice in particular. The relatively high throughput of the suggested data analysis makes application of the image data to such studies feasible. In addition to providing parameters amenable to statistical analysis, the morphometric parameters could potentially serve as inputs to hemodynamic models which can be used to evaluate the functional significance of structural changes or aberrant phenotypes ${ }^{23}$. Future studies will determine the sensitivity of the parameters for detecting and quantifying remodeling and biomechanical changes that occur in hypertensive pulmonary arterial trees.

\section{ACKNOWLEDGEMENTS}

Supported by NHLBI HL-19298, the Whitaker Foundation, the Veterans Administration, the W.M. Keck Foundation and the Falk Medical Trust.

\section{REFERENCES}

1) Higenbottam, T, "Pathophysiology of Pulmonary Hypertension--A Role for Endothelial Dysfunction", Chest 105(2):7S-12S, 1994.

2) Schultze, AE and Roth, RA, "Chronic Pulmonary Hypertension--the Monocrotaline Model and Involvement of the Hemostatic System", Journal of Toxicology and Environmental Health, Part B $1: 271-346,1998$.

3) Reid, LM, "Hypoxia and Hyperoxia: Patterns in Vascular Remodeling and Cell Adaptation", In: Pulmonary Blood Vessels in Lung Disease, Widimsky, $J$ and Herget, $J$ (eds.), Karger, Basel, 1990, pp.12-28.

4) Rabinovitch, M, Gamble, W, Nadas, AS, Miettinen, OS, and Reid, L, "Rat Pulmonary Circulation After Chronic Hypoxia: Hemodynamic and Structural Features", American Journal of Physiology 236 (Heart Circ. Physiol. 5):H818-H827, 1979.

5) Meyrick, B, Gamble, W, and Reid, L, "Development of Crotalaria Pulmonary Hypertension: Hemodynamic and Structural Study", American Journal of Physiology 239 (Heart Circ. Physiol. 8):H692-H702, 1980.

6) Jones, R and Reid, L, "Vascular Remodelling in Clinical and Experimental Pulmonary Hypertensions", In: Pulmonary Vascular Remodelling, Bishop, JE, Reeves, JT and Laurent, GJ (eds.), Portland Press, London, 1995, pp.47-115. 
7) Basu, S, Datta, BN, and Khandelwal, N, "Morphologic Changes in Pulmonary Vasculature with Arteriographic Correlation", Angiology 47(4):375-80, 1996.

8) Yen, RT, Fung, YC, and Bingham, N, "Elasticity of Small Pulmonary Arteries in the Cat", Joumal of Biomechanical Engineering 102(2): 170-7, 1980.

9) Al-Tinawi, A, Madden, JA, Dawson, CA, Linehan, JH, Harder, DR, and Rickaby, DA, "Distensibility of Small Arteries of the Dog Lung", Journal of Applied Physiology 71(5):1714-22, 1991.

10) Johnson, RH, Hu, H, Haworth, ST, Cho, PS, Dawson, CA, and Linehan, JH, "Feldkamp and Circle-and-line Conebeam Reconstruction for 3D Micro-CT of Vascular Networks", Physics in Medicine and Biology 43(4):929-40, 1998.

11) Johnson, RH, Karau, KL, Molthen, RC, and Dawson, CA, "Exploiting Self-similarity of Arterial Trees to Reduce the Complexity of Image Analysis", SPIE Volume 3660, Physiology and Function from Multidimensional Images, Clough, AV and Chen, C-T (eds.), San Diego, February 20-26, 1999, pp 351-361.

12) Johnson, RH, Hsieh, J, Molthen, RC, and Dawson, CA, "Efficacy of Beam-hardening Correction for Vascular Morphometry Using 3D Conebeam Micro-CT", Proceedings of the 1999 International Meeting on Fully Three-dimensional Image Reconstruction in Radiology and Nuclear Medicine, Egmond aan Zee, the Netherlands, June 23-26, 1999, pp 208-211.

13) Feldkamp, LA, Davis, LC, and Kress, JW, "Practical Cone-beam Algorithm", Joumal of the Optical Society of America 1(6):612-9, 1984.

14) Giuntini, C, Maseri, A, and Bianchi, R, "Pulmonary Vascular Distensibility and Lung Compliance as Modified by Dextran Infusion and Subsequent Atropine Injection in Normal Subjects", Journal of Clinical Investigation 45(11):1770-89, 1966.

15) Madden, JA, Al-Tinawi, A, Birks, E, Keller, PA, and Dawson, CA, "Intrinsic Tone and Distensibility of in Vitro and in Situ Cat Pulmonary Arteries", Lung 174:291-301, 1996.

16) Engelberg, $J$ and BuBois, AB, "Mechanics of Pulmonary Circulation in Isolated Rabbit Lungs", American Journal of Physiology 196:401, 1959.

17) Drangova, M, Holdsworth, DW, Boyd, CJ, Dunmore, PJ, Roach, MR, and Fenster, A, "Elasticity and Geometry Measurements of Vascular Specimens Using a High-resolution Laboratory CT Scanner", Physiological Measurement 14(3):277-90, 1993.

18) Bergel, DH, "The Static Elastic Properties of the Arterial Wall", Journal of Physiology 156:445-57, 1961.

19) Yen, MRT, "Elastic Próperties of Pulmonary Blood Vessels", In: Respiratory Physiology An Analytical Approach) Chang, HK and Paiva, M (eds.), Marcel Dekker, New York, 1989, pp 53359.

20) Al-Tinawi, A, Clough, AV, Harder, DR, Linehan, JH, Rickaby, DA, and Dawson, CA, "Distensibility of Small Veins of the Dog Lung", Journal of Applied Physiology 73(5):2158-65, 1992.

21) Madden, JA, Keller, PA, Effros, RM, Seavitte, C, Choy, JS, and Hacker, AD, "Response to Pressure and Vasoactive Agents by Isolated Pulmonary Arteries from Monocrotaline-treated Rats", Journal of Applied Physiology 76(4):1589.93, 1994.

22) Madden, JA, Keller, PA, Choy, JS, Alvarez, TA, and Hacker, AD, "L-Arginine-related Responses to Pressure and Vasoactive Agents in Monocrotaline-treated Rat Pulmonary Arteries", Joumal of Applied Physiology 79(2):589-93, 1995.

23) Dawson, CA, Krenz, GS, Karau, KL, Haworth, ST, Hanger, C, and Linehan, JH, "StructureFunction Relationships in the Pulmonary Arterial Tree", Journal of Applied Physiology 86(2):56983, 1998. 